\title{
Simultaneous investigation of thermal, acoustic, and magnetic emission during martensitic transformation in single-crystalline $\mathbf{N i}_{2} \mathbf{M n G a}$
}

\author{
László Z. Tóth, Lajos Daróczi, Sándor Szabó," and Dezső L. Beke \\ Department of Solid State Physics, University of Debrecen, H-4010 Debrecen, P.O. Box 2, Hungary \\ (Received 14 January 2016; revised manuscript received 12 February 2016; published 11 April 2016)
}

\begin{abstract}
Simultaneous thermal, acoustic, and magnetic emission (AE and ME) measurements during thermally induced martensitic transformation in $\mathrm{Ni}_{2} \mathrm{MnGa}$ single crystals demonstrate that all three types of the above noises display many coincident peaks and the same start and finish temperatures. The amplitude and energy distribution functions for $\mathrm{AE}$ and $\mathrm{ME}$ avalanches satisfy power-law behavior, corresponding to the symmetry of the martensite. At zero external magnetic field asymmetry in the exponents was obtained: their value was larger for heating than for cooling. Application of constant, external magnetic fields (up to $B=722 \mathrm{mT}$ ) leads to the disappearance of the above asymmetry, due to the decrease of the multiplicity of the martensite variants. Time correlations (i.e., the existence of nonhomogeneous temporal processes) within AE as well as ME emission events are demonstrated by deviations from the uncorrelated behavior on probability distributions of waiting times as well as of a sequence of number of events. It is shown that the above functions collapse on universal master curves for cooling and heating as well as for AE and ME noises. The analysis of the existence of temporal correlations between AE and ME events revealed that at short times the acoustic signals show a time delay relative to the magnetic one, due to the time necessary for the propagation of the ultrasound. At intermediate times, as expected, the magnetic signal is delayed, i.e., the magnetic domain rearrangement followed the steps of structural transformation. At much longer times the deviation from an uncorrelated (Poisson-type) behavior is attributed to the nonhomogeneity of the avalanche statistics.
\end{abstract}

DOI: 10.1103/PhysRevB.93.144108

\section{INTRODUCTION}

Ferromagnetic shape memory alloys (SMAs), like $\mathrm{Ni}_{2} \mathrm{MnGa}$, are widely used in different applications [1-5], taking advantage of the fact that in addition to their superplastic behavior with fast and large relative deformations, they can also be controlled by the application of external magnetic field. In these materials during martensitic transformation there is a strong interaction between changes of structural and magnetic domains due to the magnetoelastic coupling. Thus the jerky character of the austenite/martensite transformation is also associated with acoustic and magnetic avalanches [6-11]. The jerky magnetic signals are similar in shape to the classical (magnetic field induced) Barkhausen noise, but they have a different origin: they are initiated by the phase transformation between the two structures with different magnetization $[6,9]$ or by the rearrangement of the martensite variant structure in the martensitic state [10]. This phenomenon is called magnetic Barkhausen noise [9] or denoted as "magnetization transition spectra" [6,7], but we prefer to name it simply magnetic emission (ME), expressing the similarity to acoustic emission (AE), which also can be evoked by phase transformation [11], plastic deformation [12,13], rearrangement of martensite variant structure [14], fracture [15], etc.

Simultaneous investigations of thermal, acoustic, and magnetic noises can provide important information about the energy release/absorption, relaxation of the elastic energy by $\mathrm{AE}$, and its accompanying $\mathrm{ME}$ during austenite/martensite phase transformation (see e.g., Refs. [7,9,16-18]). Regarding

\footnotetext{
*Also at Department of Physics and Production Engineering, College of Nyíregyháza, Hungary.
}

such type of investigations during martensitic transformations there are only two recent publications in $\mathrm{Ni}_{2} \mathrm{MnGa}$ alloys available in the literature [7,9], with partly contradictory results. Using differential scanning calorimetry (DSC), polarization interferometer optics (interference-contrast colloid technique), and detection of magnetic domain dynamics by the application of a pickup coil (like in the traditional Barkhausen techniques), it was obtained in $\mathrm{Ni}_{54} \cdot 35 \mathrm{Mn}_{23} \cdot 18 \mathrm{Ga}_{22} \cdot 47$ and $\mathrm{Fe}_{70} \mathrm{Pd}_{30}$ single crystals [7] that micromagnetic reconfigurations are enslaved to the structural transformation. The sequence of the structural and micromagnetic reconfiguration was different for heating and cooling: during cooling the structural transition was followed by micromagnetic rearrangements, while the sequence was reversed for heating. On the other hand, it was underlined in Ref. [9] that, although the results of Ref. [7] seemed to indicate that the magnetic changes followed the structural ones (during cooling), ME events occurred at temperatures below the transformation temperatures (determined from the DSC results) suggesting that there was no coupling between the structural changes and ME effects. This contradiction was resolved in Ref. [9] by mentioning that in Ref. [7] the width of the structural transition was probably underestimated since the DSC was done only on a small portion of sample used for the ME measurements. Thus, using simultaneous measurements of DSC, $\mathrm{AE}$, and ME during martensitic transition on the same sample of $\mathrm{Ni}_{50} \cdot 5 \mathrm{Mn}_{29} \cdot 5 \mathrm{Ga}_{20} \cdot 0$ single crystal [9], it was demonstrated that ME indeed occurred during the structural transition and overlapping of the calorimetric, $\mathrm{AE}$, and ME activity curves was observed. Furthermore, the ME activity, both during heating and cooling, was concentrated in the lower part of the temperature range of the transition reflected by the DSC and AE results. Thus the following explanation was offered [9]: during cooling first there are 
a number of structural changes (nucleation of martensitic domains) resulting in thermal and acoustic signals. After enough changes the magnetic domains start to move giving ME signals. The process is reversed during heating: there is a simultaneous reorganization of magnetic domains and structural transformation (nucleation of the austenite) and the ME activity stops before the structural transformation is finished. In addition the statistical analysis of the AE and ME signals yielded power like probability decay with the following amplitude $\alpha$ exponents: $\alpha_{M E} \sim 4-5$ and $\alpha_{A E}=3.6$ for heating and $\alpha_{A E}=4.9$ for cooling. The values of $\alpha_{A E}$ were unexpectedly high as compared to the value of $\alpha_{A E} \sim$ 2.9 obtained in previous $\mathrm{AE}$ measurements in $\mathrm{Ni}_{2} \mathrm{MnGa}$ single crystals with similar [19] or different compositions $\left(\mathrm{Ni}_{52} \cdot 0 \mathrm{Mn}_{23} \cdot 0 \mathrm{Ga}_{25} \cdot 0\right)[17,20,21]$.

The correlations between the $\mathrm{AE}$ and $\mathrm{ME}$ events was also investigated [9], by analyzing the $P(\delta)$ distributions of the time delays $\delta$ between consecutive $A E \rightarrow M E$ signals (i.e., the $\mathrm{AE}$ signal followed by a ME signal) as well as $\mathrm{ME} \rightarrow \mathrm{AE}$ signals and it was obtained that the above histograms were undistinguishable for large $\delta$ values, and followed the $P(\delta) \sim$ $\exp (-\delta / \lambda) / \lambda$ Poisson function, indicating the uncorrelated behavior. The data showed an excess of counts above the error bars for the $\mathrm{ME} \rightarrow \mathrm{AE}$ signals, as compared to the $\mathrm{AE} \rightarrow \mathrm{ME}$ ones, only at the very first bin of the above curves confirming that certain AE signals are slightly delayed after a previous ME pulse. It was also pointed out in Ref. [9] that the order of magnitude of the observed delay was in the range of the time needed for the propagation of ultrasound through the sample/sensing device. Thus, the observed correlation indicated that at least some of the ME events had the same origin as the AE signals slightly delayed.

The above description confirms that further investigations are desired for a better understanding of the nature of the magnetoelastic coupling and the correlations between the $\mathrm{AE}$ and ME avalanches. Thus, in this paper we report detailed experimental measurements on simultaneous detection of thermal, $\mathrm{AE}$, and ME signals in a $\mathrm{Ni}_{50} \mathrm{Mn}_{28} \cdot 5 \mathrm{Ga}_{21} \cdot 5$ singlecrystalline sample. Our main aims were the following:

(i) Investigate the coupling between the $\mathrm{AE}$ and ME noises.

(ii) Provide a set of data for the energy and amplitude exponents and check the reliability of the large values obtained in Ref. [9].

(iii) Investigate the dependence of the above exponents on the constant external magnetic field.

(iv) Statistical analysis of time correlations between the events in both ME and $\mathrm{AE}$ noises and explore the correlation between the $\mathrm{AE}$ and $\mathrm{ME}$ events.

\section{EXPERIMENT}

The single-crystalline $\mathrm{Ni}_{50} \mathrm{Mn}_{28} \cdot 5 \mathrm{Ga}_{21} \cdot 5$ sample $(1 \times$ $2.5 \times 20 \mathrm{~mm}^{3}$ ) with $10-\mathrm{M}$ martensite structure was purchased from Adaptamat Co. (Finland). The sample is ferromagnetic both in martensite and austenite phase (the Curie temperature is at about $100^{\circ} \mathrm{C}$ ).

Figure 1 shows the experimental arrangement used for the detection of $\mathrm{AE}$ and $\mathrm{ME}$ signals. The sample was inserted in an Al block, placed between the magnetic poles. The halogen

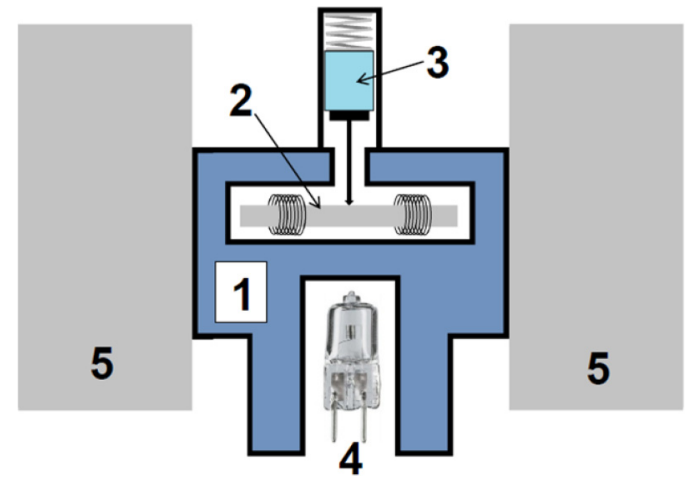

FIG. 1. Experimental arrangement. 1: Al-block; 2: sample with detector coils; 3: AE sensor with steel waveguide; 4: halogen lamp; 5: magnet poles.

lamp was used for heating. The temperature was measured with a copper-constantan thermocouple, being in close contact with the sample and controlled by a proportional-integral-derivative (PID) controller: it provided linear heating/cooling between the austenite finish $A_{f}$ and martensite finish $M_{f}$ temperatures.

Sensophone AED-404 Acoustic Emission Diagnostic equipment (from Geréb \& Co. Technical Development Ltd, Hungary), with piezoelectric sensor (MICRO-80 from Physical Acoustics Corporation), was used for the measurement of AE. The sensor coupled to the sample surface via a 15-mm-long steel waveguide to protect it from the widely variable temperatures (the temperature of the sample can be as low as $-50^{\circ} \mathrm{C}$, or as high as $100^{\circ} \mathrm{C}$ for example, in case of other SMAs). The analog-to-digital converter (ADC) sampling rate was $16 \mathrm{MHz}$ and the setup has a band-pass filter from $30 \mathrm{kHz}$ to $1 \mathrm{MHz}$ with a $30-\mathrm{dB}$ preamplifier and a maximum 100-dB main amplifier.

The magnetic signals were measured by two symmetrical detector coils around the sample $(2 \times 200$ turns $)$, with opposite winding directions to minimize the common mode external noises. The coils are about $5 \mathrm{~mm}$ long and made from 0.05 mm-diameter copper wire. The setup has a homemade $60-\mathrm{dB}$ grounded base amplifier, with very good transmissibility in the $0 \mathrm{~Hz}$ to $200 \mathrm{kHz}$ frequency range. The signals were recorded using a National Instruments PCI-6111 multifunction data acquisition board, with a 5-MS/s/channel sampling rate. All parts of the setup were grounded precisely to minimize the noises from the PID controller and the electromagnet. These measurement settings are based on our many years of experience in magnetic noise measurements [6,22]. Optimal Wiener deconvolution [23] was used to decrease the noise level in the magnetic signals, thereby getting a larger range of avalanche sizes. For simultaneous detection of $\mathrm{AE}$ and $\mathrm{ME}$ signals, we used the NI PCI-6111 data acquisition board in two-channel mode (instead of the Sensophone AED-404). The heating/cooling rate in the simultaneous $\mathrm{AE}$ and $\mathrm{ME}$ measurements was $0.06^{\circ} \mathrm{C} / \mathrm{min}$.

For DSC measurements we used a Perkin-Elmer DSC7 probe with modern control electronics, made by MRG-Info Lp. We can measure acoustic emission simultaneously with the DSC using a special accessory, coupling the AE sensor (MICRO-100s from Physical Acoustics Corporation) to the sample surface similarly, as shown in Fig. 1. The heating/ 
cooling rate was also $0.06^{\circ} \mathrm{C} / \mathrm{min}$. The $\mathrm{DSC}+\mathrm{AE}$ and $\mathrm{ME}+\mathrm{AE}$ measurements were performed on different samples: the composition and the martensite structure were the same, but the sample used for DSC + AE measurements had smaller size $\left(1 \times 2.5 \times 4.5 \mathrm{~mm}^{3}\right)$.

The classical threshold-based method was used for the identification of the magnetic avalanches: the magnetic avalanche starts when the absolute value of the signal is higher than the threshold level and finishes when it goes under the threshold. Regarding the $\mathrm{AE}$, each of the acoustic events consists of oscillating peaks with decreasing amplitude. Thus, we used the usual definition: the acoustic event starts when the first oscillation crosses the threshold (of $38 \mathrm{~dB}$ ) and finishes when the amplitude of the oscillation falls below the threshold, longer than the preset hit detection time, chosen as 30 or $100 \mu$ s. The peak amplitude is the maximum absolute value of the voltage signal between the start and finish of the event. The energy of the $i$ th peak was calculated by using the

$$
E_{i}=\frac{1}{R} \int_{\text {start }_{i}}^{\text {finish }_{i}} V^{2}(t) d t
$$

expression, where $V^{2}(t)$ is the square of the measured signal and $R$ is an arbitrary chosen resistance, $1 \mathrm{M} \Omega$. For magnetic measurements the threshold values were about $2 \mathrm{mV}$, and the maximum amplitudes were between 0.3 and $2.5 \mathrm{~V}$ (depending on the external magnetic field). For acoustic measurements the maximum peak amplitudes were about $85 \mathrm{~dB}$.

\section{RESULTS AND DISCUSSION}

\section{A. Coincidence of the ME, AE, and DSC signals}

The simultaneous detection of $\mathrm{ME}$ and $\mathrm{AE}$ as well as $\mathrm{AE}$ and DSC signals enables us to compare the three phenomena. Figures 2(a) and 2(b) show the acoustic and magnetic activity (number of hits per $1 \mathrm{~s}$ ) measured simultaneously with $0.06^{\circ} \mathrm{C} / \mathrm{min}$ rate during heating and cooling, respectively. The ME and $\mathrm{AE}$ have the same start and finish temperatures, although the magnetic activity is not proportional to the acoustic activity, and there are several unmatched peaks, where only AE or ME can be observable. This can be due to the fact that the AE sensor is sensitive only to the out-of-plane acoustic vibrations and the magnetic detector coil is sensitive only to the magnetic avalanches originating near the sample surface, not deeper than the skin depth.

Furthermore, simultaneous measurement of AE and DSC showed that the AE and DSC activities have similarly good coincidence, i.e., the start and finish temperatures are the same and most of the AE and DSC peaks overlap with each other [see Figs. 3(a) and 3(b) for illustration). Nevertheless, the finish temperatures of the $\mathrm{AE}$ and $\mathrm{ME}$, as compared to the ones dictated by the DSC results, are not well defined for cooling: there is still some, but lower level, magnetic and acoustic activity [about 5-10 hits/s; see the inset of Fig 3(b)] after the martensite finish temperature $M_{f}$. This kind of activity was also observed in previous investigations (see, e.g., Refs. [9] and [24]) and can be due either to some remaining austenite nuclei [9] or to stress relaxations inside the freshly formed martensite variant structure [24]. Note that this kind of noise activity depends on the external magnetic field too. At higher magnetic
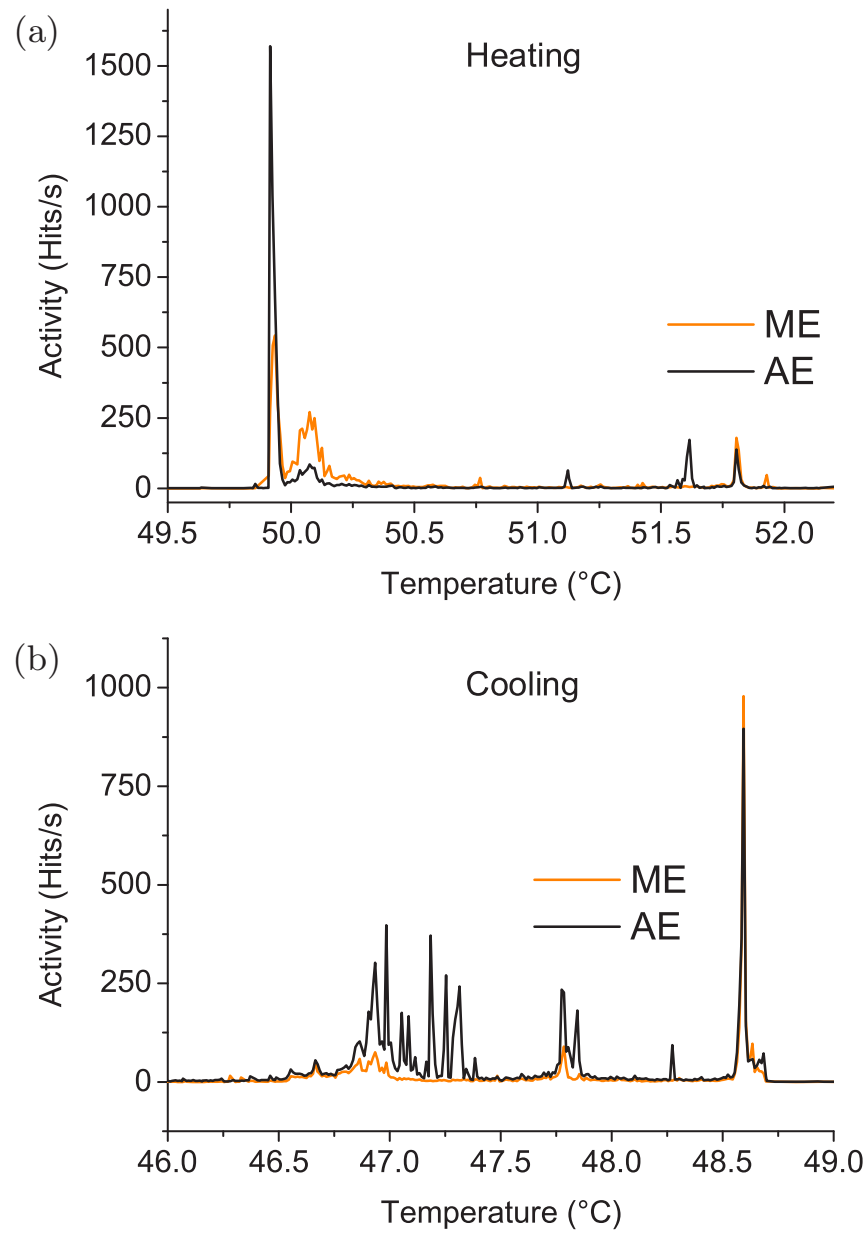

FIG. 2. Magnetic and acoustic activity during heating (a) and cooling (b) with rate $0.06^{\circ} \mathrm{C} / \mathrm{min}$.

fields, the above lower level activities were not observed below $M_{f}$.

Thus on the basis of the above observations, we can conclude that all three phenomena are in plausible coincidence with each other. In this sense, we confirm the conclusions of Ref. [9], namely that the calorimetry and AE provide the same thermal transition range.

Regarding the second statement of Ref. [9] (i.e., the $\mathrm{AE}$ and ME activity occurred in the low-temperature half segment of the transition indicated by thermal signals) one cannot get a similar conclusion from a simple look at Figs. 2 and 3. In order to get an unequivocal conclusion we plotted the acoustic activity as the function of the martensite volume fraction $\eta$ (estimated from the hysteresis loop constructed from the DSC data), for heating and cooling in Fig. 4. As it can be seen, indeed the AE activity is stronger at larger values of $\eta$ for cooling: the activity is visibly higher in the $\eta=0.5-1$ interval. On the other hand such an asymmetry is much more moderate for heating: it is small in the 0 $0.25-\eta$ interval and almost constant between 0.25 and 1 . This behavior can be related to the characteristics of the AE; during martensitic transformations the following acoustic (and magnetic) emission sources are operative (see also Refs. [24] and [25]): (i) frictional interactions of the moving interface (nucleation, pinning-depinning events)—it is active in both 

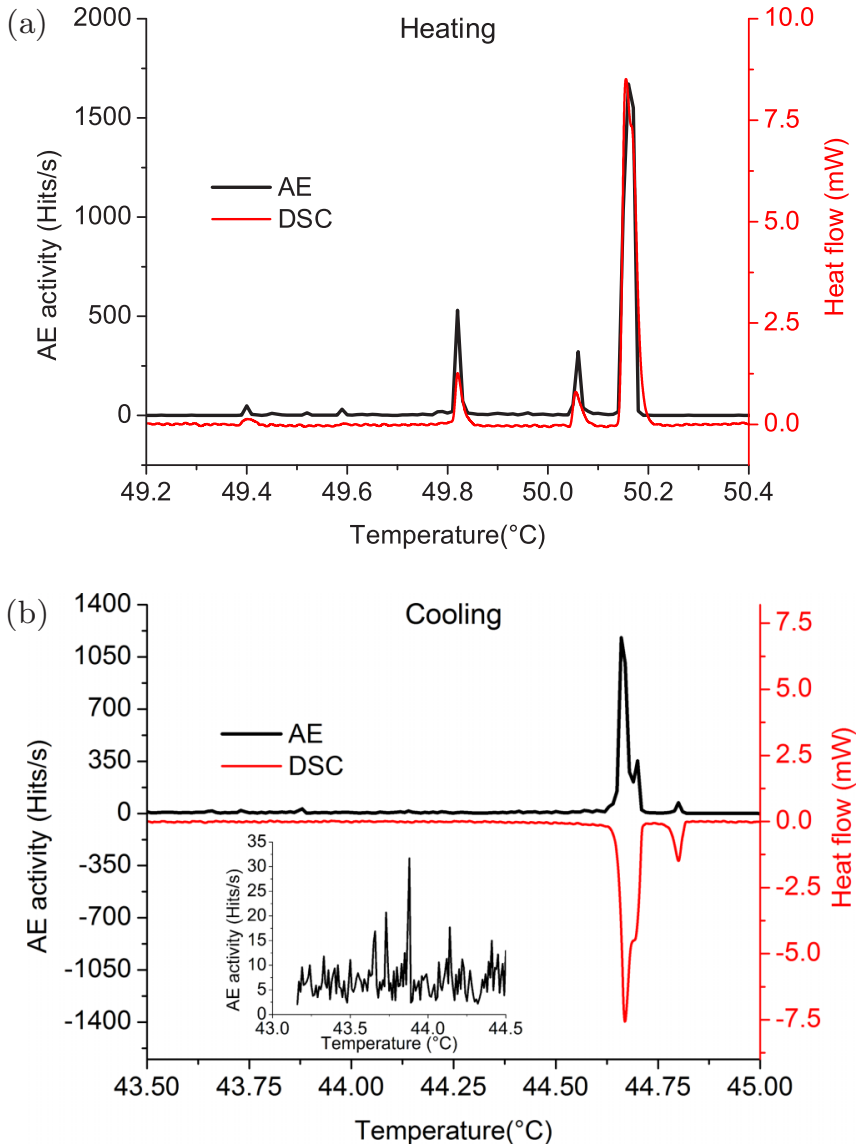

FIG. 3. Acoustic activity and DSC during heating (a) and cooling (b) with rate $0.06^{\circ} \mathrm{C} / \mathrm{min}$. The inset of (b) shows the enlarged part of acoustic activity below the DSC $M_{f}\left(\approx 44.63^{\circ} \mathrm{C}\right)$ temperature.

directions; (ii) during cooling partial relaxations of the stored elastic energy (corresponding to the total deformation strain) can occur, in the form of AE when, e.g., variants of the freshly formed martensite meet each other, as well as during heating

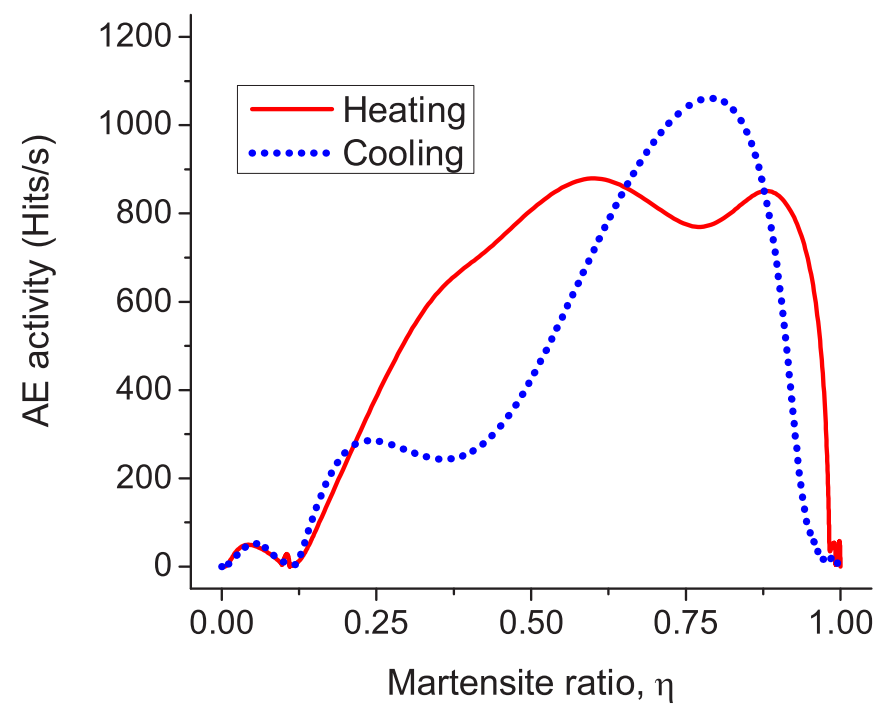

FIG. 4. Acoustic activity as function of martensite ratio $\eta$ from simultaneous AE and DSC measurements during heating and cooling. the actually stored elastic energy, accumulated and not relaxed during the forward transformation, can partly be released by AE.

Thus during cooling and heating, since the amounts of the elastic energy relaxed by AE are different, the details of the second type of emission can be different and can lead to the results shown in Fig. 4. Note that this interpretation can lead to different activity versus transformed fraction functions, depending on the material (i.e., on the type of the martensite and whether the experiments are carried out in single- or polycrystalline samples).

\section{B. Critical exponents}

As an illustration, the probability distribution function of the peak energies from ME measurement during cooling ( ${ }^{\circ} \mathrm{C} / \mathrm{min}$ ) at $B=19 \mathrm{mT}$ is shown in Fig. 5. The probability distribution functions for peak amplitudes and energies of the acoustic and magnetic signals have similar character and they can be described by the well-known power function

$$
P(X)=C X^{-\alpha} \exp \left(\frac{-X}{X_{c}}\right)
$$

Here $C$ is the normalization constant, $X$ is the corresponding noise parameter, and $X_{C}$ is the cutoff value. The exponents will be denoted by $\alpha$ and $\varepsilon$, for the amplitude and energy, respectively.

The most sophisticated method for estimating the exponent of a power function is the maximum likelihood (ML) method [26] because it is independent of the logarithmic binning, and it has only one free parameter, the minimum value of the data. Increasing the minimum value of the ML, there exists an interval within which the exponent becomes independent of the minimum value, and thus the ML method gives the right exponent. But, unfortunately the ML method cannot handle the exponential cutoff. Thus if the $X_{C}$ value is relatively low, the ML estimation cannot be applied. In our case, for the ME data, the $X_{C}$ value is often low enough to get inaccurate exponents by this method. Therefore, after logarithmic binning $\left(10^{5}-10^{6}\right.$ hits in $40-45$ bins) we made a three parametrical nonlinear fitting of the probability distribution functions, using

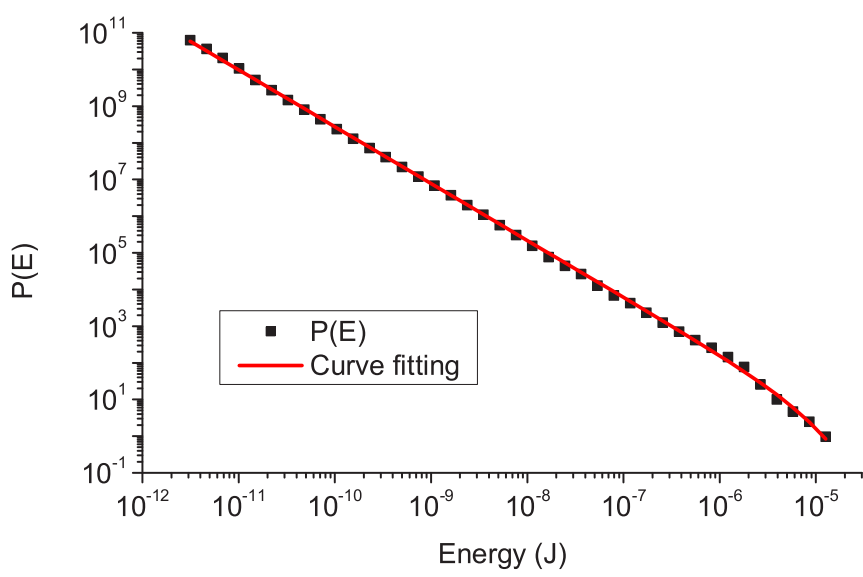

FIG. 5. Energy probability distribution function from ME at $B=$ $19 \mathrm{mT}$, during cooling $\left(2^{\circ} \mathrm{C} / \mathrm{min}\right) . P(E)=8.6 \times 10^{-8} \times E^{-1.55} \times$ $\exp \left(-E / 9 \times 10^{-6}\right)$. 

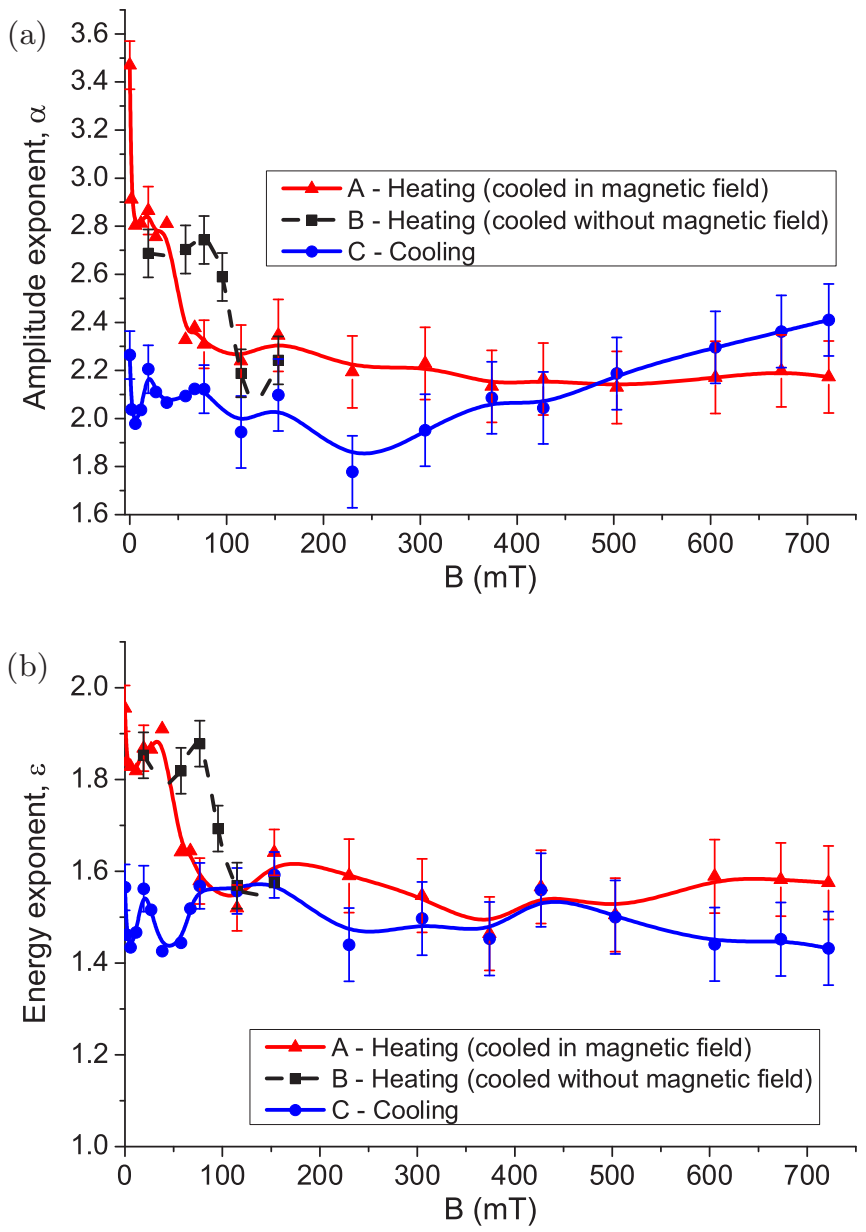

FIG. 6. ME amplitude (a) and energy (b) exponents as function of magnetic field.

the Levenberg-Marquadt least-square method (see the example in Fig. 5). The probability distribution function is linear on a $\log$-log scale over many orders of magnitude, indicating that the fitting gives the correct exponent with small fitting errors.

Figure 6 shows the magnetic field dependence of the $\alpha_{M E}$ (a) and $\varepsilon_{M E}$ (b) exponents, for the following:

(A) heating $(M \rightarrow A)$, the preceding $A \rightarrow M$ transformation was performed in the same magnetic field,

(B) heating $(M \rightarrow A)$, the preceding $A \rightarrow M$ transformation was performed in zero magnetic field,

(C) cooling $(A \rightarrow M)$.

It can be seen that there is a decrease in the critical exponents $\alpha_{M E}$ and $\varepsilon_{M E}$ : at about $50 \mathrm{mT}$ as well as at $100 \mathrm{mT}$ for cases (A) and (B), respectively, during heating, while for cooling there is no considerable field dependence. The $\alpha_{A E}$ and $\varepsilon_{A E}$ exponents from the acoustic emission measurements behave similarly [Figs. 7(a) and 7(b).

Indeed, similar values of the exponents and their field dependence for AE was observed in Refs. [17,19], and [20] during martensitic transformation in similar single-crystalline samples, as well as for the ME critical exponents during plastic deformation induced variant rearrangement in martensite state [10]. This was interpreted $[10,11]$ by the decrease of the multiplicity of the martensite variants as follows: if the external field exceeds the switching field necessary to move the twin
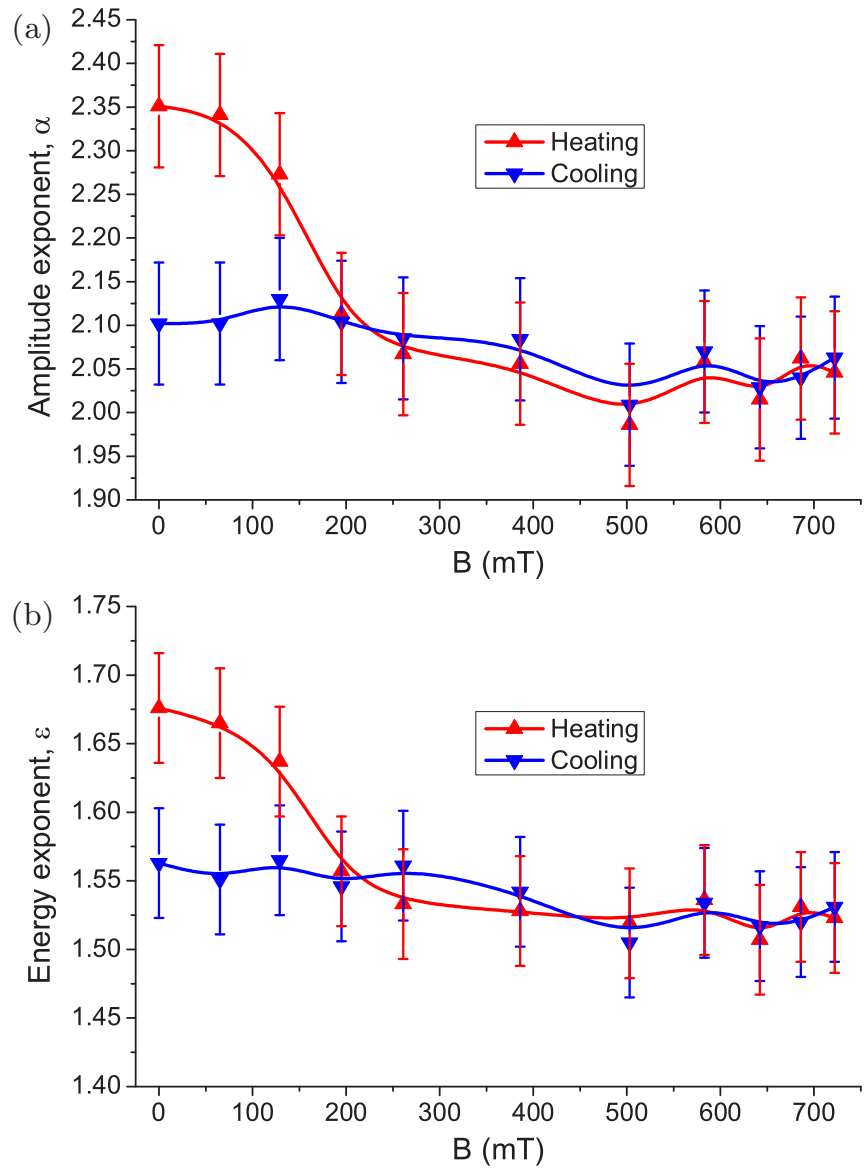

FIG. 7. AE amplitude (a) and energy (b) exponents as function of magnetic field (the curves for heating correspond to the case when the preceding cooling was made in magnetic field: see curves A in Fig. 6).

boundaries, the twin variants can go to large sizes before their collisions, since the external field selects a restricted (small) number of growth directions of martensite plates. Thus above the threshold value fewer number of interactions dissipate higher energy and thus, e.g., the energy distribution will have a smaller slope.

It is interesting that while the drop for both the energy and amplitude exponents shown in Figs. 6(a) and 6(b) for ME is similar to the drop in the above cited cases (about 25\%), the drop is a bit less for AE [Figs. 7(a) and 7(b)] for heating. We did not observe any considerable change in the exponents for cooling. Note that in Ref. [19] the drop is a bit more moderate for cooling (see Fig. 3 in Ref. [19]), while in Ref. [20] only the results for heating are determined. In addition, the switching field in Refs. [19] and [20] was about 0.6 T, while here we see the drop is at about $0.1-0.2 \mathrm{~T}$, which is in good accordance with the value obtained in Ref. [10] for the same sample composition. The difference in the change of the threshold value for the samples cooled with and without magnetic field can be interpreted similarly by the decrease of the multiplicity; during cooling in zero field multivariant martensite structure develops, while heating in increasing magnetic field the variant structure becomes more and more ordered approaching the monovariant structure. As a consequence the drop in the 
exponents starts at smaller field if the sample was cooled in the same magnetic field in which it was heated [Figs. 6(a) and $6(\mathrm{~b})]$.

Our energy and amplitude exponents for both AE and ME are much smaller than those obtained in Ref. [9]. Although the composition of samples measured in Ref. [9] were different, we believe that the large values are less reasonable and values close to the ones obtained by us and in Refs. [17,19], and [20] are expected on the basis of the range of existence for such exponents with different martensite symmetry (see Table 1 in Ref. [11]). The nice correspondence of the acoustic and magnetic signals suggests that the acoustic and magnetic exponents can be similar. As it is illustrated in Figs. 6(b) and 7(b), at higher magnetic fields, where monovariant martensite structure develops, indeed the acoustic and magnetic energy exponents have the same value (1.55) and there is no difference between heating and cooling.

The observed asymmetry in the critical exponents during cooling and heating in both the $\mathrm{AE}$ and $\mathrm{ME}$ at zero magnetic field is similar to the observations published in Refs. [17] and [18] for AE during thermally induced transformations and to the results of Ref. [27] obtained during strain field induced cubic/monoclinic transition in strain intermittency of $\mathrm{CuAlBe}$ single crystals.

The well-known scaling rule [11], $(\alpha-1) /(\varepsilon-1)=z$, with $z \cong 2$ is valid for all the $\alpha$ and $\varepsilon$ exponents, with a clear exception for ME above $300 \mathrm{mT}$. Since in this case the $\alpha$ versus $B$ as well as the $\varepsilon$ versus $B$ functions during cooling are slightly ascending as well as descending, respectively (Fig. 6), the value of $z$ gradually deviates from 2 and its value is about 3 at $720 \mathrm{mT}$.

\section{Correlations}

We can investigate two types of time correlations. First, like it was done in Ref. [28], one can check whether there exists any interdependence between closely recorded signals or not by creating the waiting time distributions $P(\tau)$, with $\tau=$ $t_{k+1}-t_{k}$, the waiting time or the time between consecutive jerks. It was shown [28-31] that the waiting time probability density fulfills a scaling law

$$
P(\tau)=\frac{1}{\langle\tau\rangle} \Phi(\tau /\langle\tau\rangle),
$$

where $\langle\tau\rangle$ is the mean waiting time and $\Phi$ is a universal function of its argument.

It can be seen in Fig. 8 that the normalized probability distribution functions of waiting times both for $\mathrm{AE}$ and $\mathrm{ME}$ at $B=0 \mathrm{~T}$ deviate from the Poisson-type behavior,

$$
P(\tau)=\lambda \exp (-\tau \lambda)
$$

(where $\lambda=1 /\langle\tau\rangle$ is the Poissonian rate). It can be characterized by two power functions with $-(1-v)=-0.95$ and $-(2+\xi)=-2.05$ for small and large arguments, respectively. These results suggest rather similar statistical behavior as obtained in polycrystalline $\mathrm{Cu}_{67} \cdot 64 \mathrm{Zn}_{16} \cdot 71 \mathrm{Al}_{15} \cdot 65$ shape memory alloy from $\mathrm{AE}$ and calorimetric signals in Ref. [28] (see Fig. 5 therein): deviations from the uncorrelated Poisson behavior are due to Omori-like correlations (after shock effects) for small arguments with exponent $-(1-v)=$

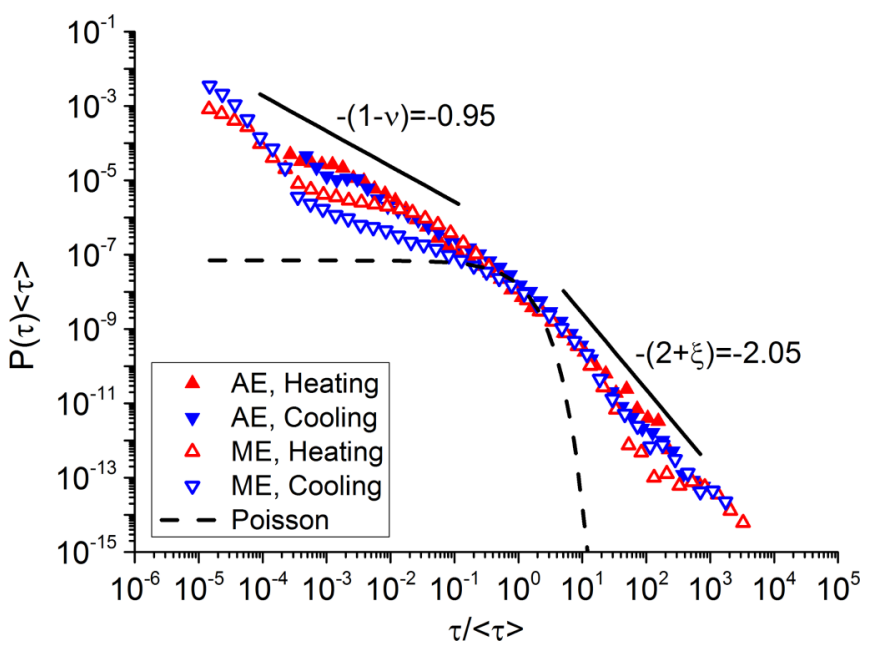

FIG. 8. Probability distribution function of waiting times $\tau$ for acoustic and magnetic events at $B=0 \mathrm{~T}$. The horizontal and vertical axes are normalized by $1 /\langle\tau\rangle$ and $\langle\tau>$ respectively, where $\langle\tau\rangle \approx 0.1-0.5 \mathrm{~s}$ is the mean waiting time. The dashed (Poisson) line corresponds to $\lambda=1.5 \mathrm{~s}^{-1}$.

-0.9 , while for large arguments to temporal nonhomogeneity of the avalanches with an exponent $-(2+\xi)=-2.2$. It was also shown in Ref. [28] that the latter value was the result of the double Gaussian function of the activity versus temperature [as contrasted to a single Gaussian evolution, leading to exponential decay given by (4)]. Indeed. it can be seen that our activity versus temperature curves (see Figs. 2 and 3) are not single Gaussian functions.

It was pointed out recently [32] that if temporal correlations exist in the sequences of discrete events (inhomogeneous temporal processes) - as suggested by the power-law behavior of the $\Phi$ function for large arguments-it is also useful to make comparison between the experimentally determined $P\left(n ; \tau_{m}\right)$ functions with the $P_{\text {ind }}\left(n ; \tau_{m}\right)$ distributions

$$
P_{\text {ind }}\left(n ; \tau_{m}\right)=a^{n-1}(1-a),
$$

belonging to a sequence of $n$ successive independent events within a burst [12]. Here $a\left(\tau_{m}\right)=\int_{0}^{\tau_{m}} P(\tau) d \tau$ and $\tau<\tau_{m}$, where $\tau_{m}$ is the maximum time difference between subsequent events in a burst. The authors of Ref. [12] used the word "burst" as a long sequence of AE events: $\tau_{m}$ was typically about two orders of magnitude longer than the characteristic time for a single event, $0.1-10 \mathrm{~ms}$. For independent events $a^{n-1}$ is the probability that $n-1$ events follow the first event with $\tau<\tau_{m}$ and $(1-a)$ is the probability that for the subsequent event $\tau>$ $\tau_{m}$ [12]. Figure 9 shows the $P\left(n ; \tau_{m}\right)$ functions of the acoustic and magnetic events at $B=0 \mathrm{~T}$ for heating and cooling with, similarly as was taken in Ref. [12], $\tau_{m}=0.5 \mathrm{~s}$. The $P\left(n ; \tau_{m}=\right.$ $0.5 \mathrm{~s})$ distributions follow power-law behavior, $P(n) \sim n^{\beta}$, with $\beta=-1.9$ exponent. The dashed line indicates $P_{\text {ind }}\left(n ; \tau_{m}\right)$ [Eq. (5)] with $a=0.6$ for independent events. Our result is similar to Fig. 6 of Ref. [12] where a similar analysis was carried out for AE data obtained during torsional deformation of bulk metallic glasses with $\beta=-2.0$. Thus, similarly as in Ref. [12], we can conclude that the $P\left(n ; \tau_{m}\right)$ distributions are clearly different from $P_{\text {ind }}\left(n ; \tau_{m}\right)$, demonstrating that correlations exist between the acoustic events as well as 


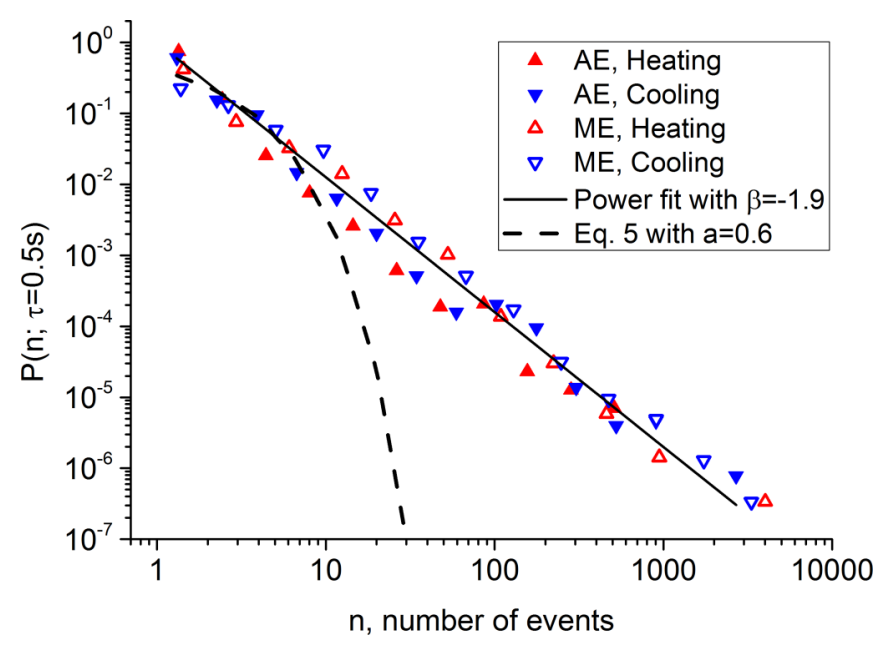

FIG. 9. Probability distribution of a sequence of $n$ acoustic and magnetic events at $B=0 \mathrm{~T}$, belonging to the same burst with $\tau<$ $\tau_{m}=0.5 \mathrm{~s}$. Dashed line shows Eq. (5) with $a=0.6$ and the solid line shows the power-law behavior with $\beta=-1.9$.

between the magnetic events. This conclusion is in line with the one deduced above from the power-law behavior of the $P(\tau)\langle\tau\rangle=\Phi(\tau /\langle\tau\rangle)$ function for large arguments.

Before turning to the investigation of the correlation between the ME and AE signals, it is worth emphasizing that in Figs. 8 and 9 the statistical characteristics of both types of signals are the same and the functions are also scaled together for cooling and heating, confirming the universal character of these functions.

The simultaneous measurement of the $\mathrm{AE}$ and $\mathrm{ME}$ also enables us to study the correlation between these two signals in detail, similarly as was done in Ref. [9]. Let $\delta_{M E \rightarrow A E}$ indicate the time delay between consecutive signals, when a magnetic avalanche is followed by an acoustic one, and $\delta_{A E \rightarrow M E}$ the time delay, when an acoustic event is followed by a magnetic one. If the two signals are correlated, the two types of delays should have different probability distributions. For uncorrelated signals, the two delays have the same distribution, which can be described by the Poisson function given by relation (4).

Figures 10(a) and 10(b) show the probability density function of the delays for heating and cooling with $0.06^{\circ} \mathrm{C} / \mathrm{min}$ heating/cooling rate at zero external magnetic field and at $B=600 \mathrm{mT}$, respectively. At intermediate values of $\delta$, the $\mathrm{AE}$ and ME events seems uncorrelated; the probability functions are close to the exponential function with $\lambda \approx 150 \mathrm{~s}^{-1}$. As can be seen, even at higher values of $\delta$, there are some deviations from the Poisson function which, similarly as in Ref. [9], can be explained by the variation of the rate during the transition (nonhomogeneous character of the random process [29]) as discussed also before. What is more interesting, below $\delta=10^{-4}$ s the $P\left(\delta_{M E \rightarrow A E}\right)$ and $P\left(\delta_{A E \rightarrow M E}\right)$ curves are diverging, indicating that in the $\delta=10^{-5}-10^{-4}$-s range it is more probable that an acoustic signal is followed by a magnetic one, than inversely. This conclusion contradicts the results of Ref. [9] where the sequence of the two signals was the opposite only in one point, at $\delta=10^{-4} \mathrm{~s}$. On the other hand it is in accordance with the plausible expectation, that the acoustic
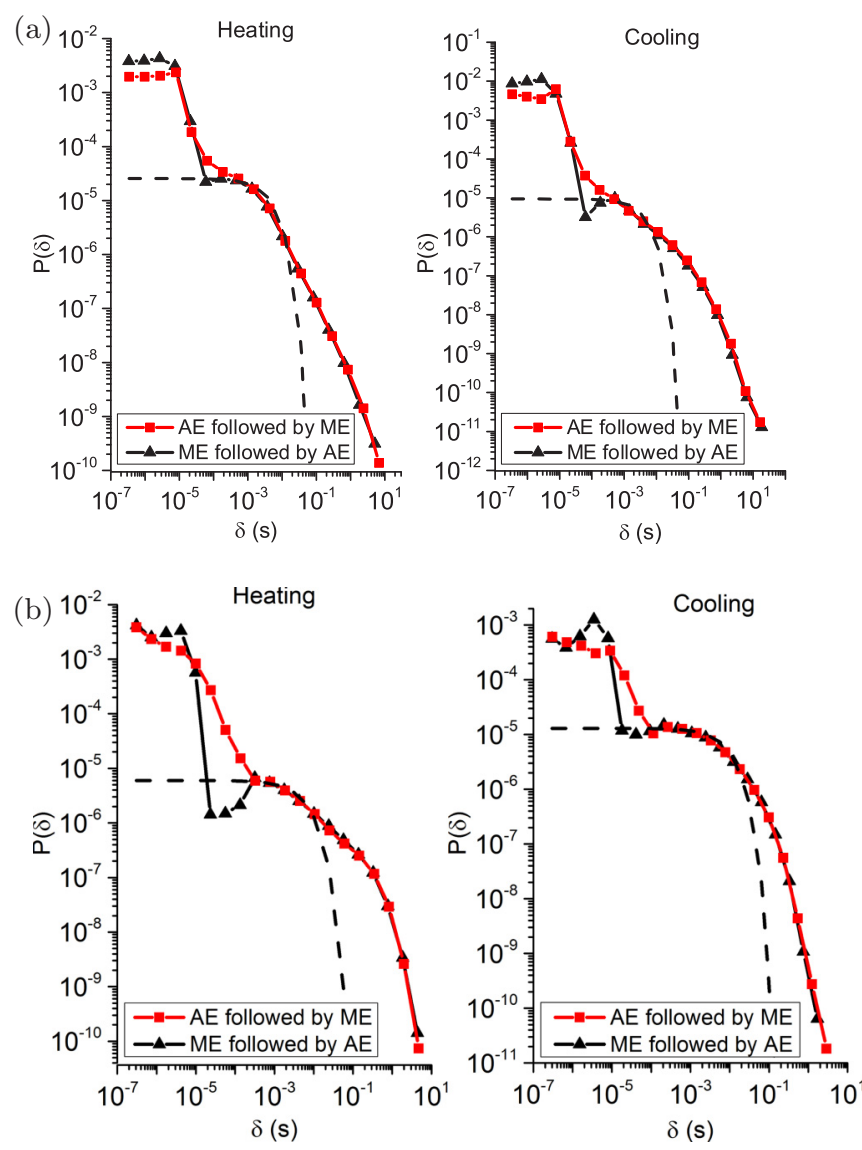

FIG. 10. Probability density function of the $\delta_{A E \rightarrow M E}$ and $\delta_{M E \rightarrow A E}$ delays for heating and cooling with $0.06^{\circ} \mathrm{C} / \mathrm{min}$ heating/cooling rate at $B=0 \mathrm{mT}$ (a) and $B=600 \mathrm{mT}$ (b). The dashed lines indicate the exponential function for uncorrelated signals.

emission, accompanied with the jerky phase transition, is the primary effect, and the magnetic domain rearrangement occurs as a consequence of the structural transformation. Below about $\delta=5 \mu$ s the sequence of the two signals is the opposite i.e., it is more probable that a magnetic signal is followed by an acoustic one. This change can be due to the fact that while the electromagnetic delay (due to the time necessary for the signals to reach the detector) of the magnetic signals is negligible, the acoustic propagation delay of the acoustic signals can be comparable to the times present on the left-hand side of the time scale in Figs. 10(a) and 10(b). In fact this is the same argument as used in Ref. [9] to explain their conclusion drawn, but without giving any numerical estimate for the time $\delta=10^{-4}$ s observed there. One can make such an estimation taking as a typical value $5000 \mathrm{~m} / \mathrm{s}$ for the sound velocity and using the characteristic dimension of our arrangement (size of the sample and the pickup needle) as $L \cong 25 \mathrm{~mm}$. This gives about $5 \mu \mathrm{s}$, which is about 20 times less than the value at which the correlation was observed in Ref. [9], but coincides with the $5 \mu$ s below which we obtain that the acoustic signals are delayed as compared with the magnetic ones.

Figures 10 (a) and 10(b) also show that the $P(\delta)$ functions are very similar at $0 \mathrm{mT}$ and at $600 \mathrm{mT}$ external magnetic field. The only difference is that at high magnetic field the acoustic and magnetic signals seems more correlated, because 
the differences between the $P\left(\delta_{M E \rightarrow A E}\right)$ and $P\left(\delta_{A E \rightarrow M E}\right)$ curves are more significant. This can be due to the fact that in the case of Fig. 10(b) the sample is fully magnetized and the magnetic domain rearrangement follows the steps of structural transformation more tightly.

Note that at high magnetic field, the magnetic detector coils can also detect acoustic emission, like the electromagnetic acoustic transducers (EMAT). We tested this effect experimentally in the following way: at high magnetic field we generated acoustic waves in the sample using the piezoelectric AE sensor as a wave source with a signal generator. The acoustic signals detected by the magnetic detector coils were always below the threshold level corresponding to the background noise of the system.

It is important to mention that in the evaluation of the $\mathrm{AE}$ signals the results (e.g., the value of the exponents) can be sensitive, especially at relatively large driving rates, to the so-called hit definition time and the hit lockout time (or to their sum, which can be called the hit detections time, HDT) [33]. Figures 10(a) and 10(b) correspond to HDT $=100 \mu \mathrm{s}$. As a check we plotted the same function with HDT $=30 \mu \mathrm{s}$ and the results were practically the same.

\section{CONCLUSIONS}

From simultaneous thermal, acoustic, and magnetic emission measurements during thermally induced martensitic transformation in $\mathrm{Ni}_{2} \mathrm{MnGa}$ single crystals it was obtained that they all have jerky character at the low heating rate $\left(0.06^{\circ} \mathrm{C} / \mathrm{min}\right)$. The noise activities were in good coincidence with each other (with many coincident peaks and the same start and finish temperatures).

The critical exponents for AE, characterizing the expected power-law behavior, have values in accordance with those ob- tained in Refs. [17,19], and [20] (and in line with expectations based on the symmetry of the martensite), but differ from those obtained in Ref. [9]. The nice correspondence of the AE and ME activities as well as the similar values of the critical exponents are related to the similar physical origin of the two types of noise. At the same time, a certain (and a bit different) nonuniform AE activity was observed for heating and cooling as the function of transformed martensite volume fraction $\eta$ : both were larger for larger $\eta$.

At zero external magnetic field certain asymmetry in the above exponents was also obtained: their value was larger for heating than for cooling. Application of constant, external magnetic field (up to $B=722 \mathrm{mT}$ ) led to disappearance of the above asymmetry by the decrease of the exponents belonging to heating, which was interpreted by the decrease of the multiplicity of the martensite variants.

Time correlations (i.e., the existence of inhomogeneous temporal processes) within groups of both acoustic and magnetic emission events are demonstrated by deviations from the uncorrelated behavior on probability distributions of waiting times as well as of a sequence of number of events. It is shown that the above functions collapse on universal master curves [ $\Phi(\tau /\langle\tau\rangle)$ and $P\left(n ; \tau_{m}\right)$ functions, respectively] for cooling and heating as well as for $\mathrm{AE}$ and $\mathrm{ME}$ noises.

The analysis of the existence of temporal correlations between acoustic and magnetic events revealed that at short times the acoustic signals show a time delay relative to the magnetic ones, due to the time necessary for the propagation of the ultrasound. At intermediate times, as expected, the magnetic signals are delayed, i.e., the magnetic domain rearrangement followed the steps of structural transformation. At much longer times the deviation from an uncorrelated (Poisson-type) behavior is attributed to the nonhomogeneity of the avalanche statistics.
[1] O. Heczko, N. Scheerbaum, and O. Gutfleisch, in Nanoscale Magnetic Materials and Applications, edited by J. P. Liu, E. Fullerton, O. Gutfleisch, and D. Sellmyer (Springer, New York, 2009), pp. 399-439.

[2] B. Krevet, V. Pinneker, and M. Kohl, Smart Mater. Struct. 21, 094013 (2012).

[3] K. Ullakko, L. Wendell, A. Smith, P. Müllner, and G. Hampikian, Smart Mater. Struct. 21, 115020 (2012).

[4] L. Straka, O. Heczko, H. Seiner, N. Lanska, J. Drahokoupil, A. Soroka, S. Fähler, H. Hänninen, and A. Sozinov, Acta Mater. 59, 7450 (2011).

[5] A. R. Smith, A. Saren, J. Järvinen, and K. Ullakko, Microfluid. Nanofluid. 18, 1255 (2015).

[6] Z. Balogh, L. Daróczi, L. Harasztosi, D. L. Beke, T. A. Lograsso, and D. L. Schlagel, Mater. Trans. 47, 631 (2006).

[7] M. R. Sullivan, A. A. Shah, and H. D. Chopra, Phys. Rev. B 70, 094428 (2004).

[8] J. N. Armstrong, J. D. Felske, and H. D. Chopra, Phys. Rev. B 81, 174405 (2010).

[9] J. Baró, S. Dixon, R. S. Edwards, Y. Fan, D. S. Keeble, L. Mañosa, A. Planes, and E. Vives, Phys. Rev. B 88, 174108 (2013).
[10] L. Daróczi, S. Gyöngyösi, L. Z. Tóth, S. Szabó, and D. L. Beke, Appl. Phys. Lett. 106, 041908 (2015).

[11] A. Planes, L. Mañosa, and E. Vives, J. Alloys Compd. 577, Suppl. 1, S699 (2013).

[12] Z. Kovács, M. Ezzeldien, K. Máthis, P. Ispánovity, F. Chmelík, and J. Lendvai, Acta Mater. 70, 113 (2014).

[13] I. Shashkov, M. Lebyodkin, and T. Lebedkina, Acta Mater. 60, 6842 (2012).

[14] L. Straka, V. Novak, M. Landa, and O. Heczko, Mater. Sci. Eng. A 374, 263 (2004).

[15] Z. Danku, G. B. Lenkey, and F. Kun, Appl. Phys. Lett. 106, 064102 (2015).

[16] M. C. Gallardo, J. Manchado, F. J. Romero, J. del Cerro, E. K. H. Salje, A. Planes, E. Vives, R. Romero, and M. Stipcich, Phys. Rev. B 81, 174102 (2010).

[17] L. Z. Tóth, S. Szabó, L. Daróczi, and D. L. Beke, Phys. Rev. B 90, 224103 (2014).

[18] M. K. Bolgár, L. Z. Tóth, S. Szabó, S. Gyöngyösi, L. Daróczi, E. Y. Panchenko, Y. I. Chumlyakov, and D. Beke, J. Alloys Compd. 658, 29 (2016).

[19] R. Niemann, J. Baró, O. Heczko, L. Schultz, S. Fähler, E. Vives, L. Mañosa, and A. Planes, Phys. Rev. B 86, 214101 (2012). 
[20] B. Ludwig, C. Strothkaemper, U. Klemradt, X. Moya, L. Mañosa, E. Vives, and A. Planes, Appl. Phys. Lett. 94, 121901 (2009).

[21] F. J. Pérez-Reche, E. Vives, L. Mañosa, and A. Planes, Phys. Rev. Lett. 87, 195701 (2001).

[22] G. Eszenyi, S. Szabó, L. Harasztosi, F. Zámborszky, J. Nyéki, Z. Erdélyi, and D. Beke, J. Mater. Res. 24, 130 (2009).

[23] W. H. Press, S. Teukolsky, W. Vetterling, and B. Flannery, Numerical Recipes in c: The Art of Scientific Computing, (Cambridge Univ. Press, Cambridge, 1988), pp. 547-549.

[24] J. Baram and M. Rosen, Acta Metall. 30, 655 (1982).

[25] R. Niemann, J. Kopecek, O. Heczko, J. Romberg, L. Schultz, S. Fähler, E. Vives, L. Mañosa, and A. Planes, Phys. Rev. B 89, 214118 (2014).

[26] A. Clauset, C. R. Shalizi, and M. E. Newman, SIAM Rev. 51, 661 (2009).
[27] X. Balandraud, N. Barrera, P. Biscari, M. Grédiac, and G. Zanzotto, Phys. Rev. B 91, 174111 (2015).

[28] J. Baró, J.-M. Martín-Olalla, F. J. Romero, M. C. Gallardo, E. K. H. Salje, E. Vives, and A. Planes, J. Phys.: Condens. Matter 26, 125401 (2014).

[29] P. Bak, K. Christensen, L. Danon, and T. Scanlon, Phys. Rev. Lett. 88, 178501 (2002).

[30] Á. Corral and K. Christensen, Phys. Rev. Lett. 96, 109801 (2006).

[31] J. Baró, Á. Corral, X. Illa, A. Planes, E. K. H. Salje, W. Schranz, D. E. Soto-Parra, and E. Vives, Phys. Rev. Lett. 110, 088702 (2013).

[32] M. Karsai, K. Kaski, A.-L. Barabási, and J. Kertész, Sci. Rep. 2, 397 (2012).

[33] M. A. Lebyodkin, I. V. Shashkov, T. A. Lebedkina, K. Máthis, P. Dobron, and F. Chmelík, Phys. Rev. E 88, 042402 (2013). 